Theoretical and Mathematical Physics, 170(1): 2-16 (2012)

\title{
LIGHT-CONE WAVE FUNCTIONS OF HEAVY BARYONS
}

\author{
(C) A. Ali, ${ }^{*}$ C. Hambrock,${ }^{*}$ and A. Ya. Parkhomenko ${ }^{\dagger}$
}

We present a classification of the three-quark light-cone distribution amplitudes (LCDAs) for ground-state heavy baryons with the spin parities $J^{P}=1 / 2^{+}$and $J^{P}=3 / 2^{+}$in $Q C D$ in the heavy-quark symmetry limit and calculate several lowest moments of LCDAs based on QCD sum rules. We propose simple model functions for the heavy-baryon distribution amplitudes and analyze their dependence on the energy scale.

Keywords: heavy baryon, distribution amplitude, sum rule

\section{Introduction}

After approximately a decade of operation, the $B$-meson factories at SLAC and KEK have greatly contributed to clarifying the nature of $C P$ violation in the quark sector of the standard model (SM). Studying heavy bottom baryons at the LHC can additionally test the Kobayashi-Maskawa mechanism. Specific processes with bottom baryons, such as rare decays involving flavor-changing neutral current (FCNC) transitions, are potential sources of new physics beyond the SM. In contrast to $B$ mesons, a nonzero spin of baryons also allows studying spin correlations experimentally. The spectrum of heavy bottom baryons has been substantially enlarged thanks to the efforts of the CDF and D0 collaborations at the Tevatron collider during the last several years. Despite this progress, studying FCNC-motivated decays of bottom baryons remains statistically limited. A greater success is expected at the LHC, where heavy baryons will be copiously produced and their weak decays may be measured sufficiently precisely to manifest physics beyond the SM.

The theory of bottom baryon decays into light hadrons is more complicated than the $B$-meson decays and hence received less attention. Calculations of heavy-baryon decays into light particles based on the heavy quark expansion (see, e.g., [1]) or using the QCD sum-rule method developed in [2]-[4] require the primary nonperturbative objects, the distribution amplitudes of heavy baryons. For a long time, the existing models of heavy-baryon distribution amplitudes [5], [6] were based on quark models and were not fully consistent with QCD. In [7], the complete classification of three-quark light-cone distribution amplitudes (LCDAs) of the $\Lambda_{b}$ baryon in QCD in the heavy-quark symmetry limit was given, and the energy-scale dependence of the leading-twist LCDA was analyzed. In addition, simple models of the LCDAs were suggested, and their parameters were fixed based on estimates of the first few moments by the QCD sumrule method. The analysis in [7] can be extended to all the ground-state $b$ baryons with both the spin parities $J^{P}=1 / 2^{+}$and $J^{P}=3 / 2^{+}$. We summarize the basic steps and main results of such an analysis here and present all the details in a forthcoming paper [8].

*Deutsches Elektronen-Synchrotron DESY, Hamburg, Germany,

e-mail: ahmed.ali@desy.de, hambrock@mail.desy.de.

${ }^{\dagger}$ Yaroslavl State University, Yaroslavl, Russia, e-mail: parkh@uniyar.ac.ru.

Prepared from an English manuscript submitted by the authors; for the Russian version, see Teoreticheskaya $i$ Matematicheskaya Fizika, Vol. 170, No. 1, pp. 5-21, January, 2012. 


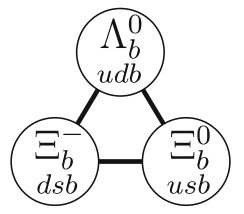

a

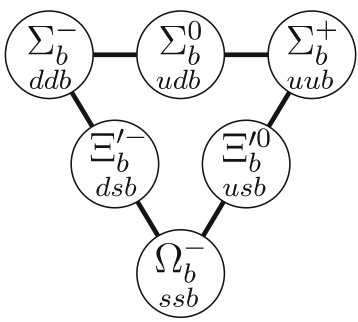

$\mathrm{b}$

Fig. 1. The $S U(3)_{F}$ flavor multiplets of bottom baryons: (a) the $S U(3)_{F}$ triplet with the spin parity $J^{P}=1 / 2^{+}$and scalar $\left(j^{p}=0^{+}\right)$light-quark state; (b) the $S U(3)_{F}$ sextet with the axial vector $\left(j^{p}=1^{+}\right)$light-quark state. The particle content in the diagram corresponds to the bottom baryons with the spin parity $J^{P}=1 / 2^{+}$. The ones with $J^{P}=3 / 2^{+}$have the $*$-modification of the names.

\section{Interpolating heavy-baryon currents}

In the heavy-quark effective theory (HQET), baryons with one heavy quark $Q=c, b$ are classified according to the angular momentum $\ell$ and parity $p$ of the light quark pair called the diquark (see [9] for a discussion of this issue). The heavy quarks are nonrelativistic particles that decouple from the diquark in the leading order of the $1 / m_{Q}$ expansion.

The ground-state baryons $(\ell=0)$ with the spin parity $J^{P}$ are characterized by the spin parity $j^{p}$ of the diquark. The spins of the light quarks produce two states with $j^{p}=0^{+}$and $j^{p}=1^{+}$. In the state with $j^{p}=0^{+}$, the spin wave function is antisymmetric, while the Fermi statistics of the baryon state and antisymmetry in color space require an antisymmetric flavor wave function. This results in a baryonic state with the isospin $I=0$ constructed from the light $u$ and $d$ quarks, which is called the $\Lambda_{Q}$ baryon (the spin parity is $J^{P}=1 / 2^{+}$). When the spin parity of the diquark is $j^{p}=1^{+}$, the spin part of the baryon wave function is symmetric, which requires symmetry of the wave function in the flavor space. In the case of only light $u$ and $d$ quarks, this gives two degenerate states with the isospin $I=1$, which are called $\Sigma_{Q}$ and $\Sigma_{Q}^{*}$ baryons and have the respective spin parities $J^{P}=1 / 2^{+}$and $J^{P}=3 / 2^{+}$. Including the $s$ quark increases the number of heavy baryons in the multiplet, which (in addition to the isospin) is characterized by the strangeness $S$. For $S=-1$, there are two baryonic states $\Xi_{Q}$ and $\Xi_{Q}^{\prime}$ with $J^{P}=1 / 2^{+}$and also the $\Xi_{Q}^{*}$ baryon with $J^{P}=3 / 2^{+}$. The $S U(3)_{F}$ multiplets of $b$-quark baryons are shown in Fig. 1.

The heavy-baryon local currents have the general structures [10], [11]

$$
J_{1}^{H_{Q}}=\varepsilon_{a b c}\left[\psi^{a \mathrm{~T}} \mathcal{C} \Gamma \mathcal{T} \psi^{b}\right] \Gamma^{\prime} Q_{v}^{c}, \quad J_{2}^{H_{Q}}=\varepsilon_{a b c}\left[\psi^{a \mathrm{~T}} \mathcal{C} \Gamma \not \mathcal{T} \psi^{b}\right] \Gamma^{\prime} Q_{v}^{c},
$$

where $a, b, c=1,2,3$ are color indices, $\psi$ is an $S U(3)_{F}$ triplet in the flavor space, $v_{\mu}$ is the four-velocity of the heavy quark, $\not b=(v \gamma), Q_{v}$ is the effective static field of the heavy quark satisfying $\not<Q_{v}=Q_{v}$, the superscript $\mathrm{T}$ denotes transposition, $\mathcal{C}$ is the charge conjugation matrix with the properties $\mathcal{C} \gamma_{\mu}^{\mathrm{T}} \mathcal{C}^{-1}=-\gamma_{\mu}$ and $\mathcal{C} \gamma_{5} \mathcal{C}^{-1}=\gamma_{5}$, and $\mathcal{T}$ is some matrix in the flavor space.

For each of the ground-state baryons, there are two independent interpolating local currents $J_{1}$ and $J_{2}$, both having the appropriate quantum numbers. They can be constructed as suggested in [10]-[13]. For the states belonging to the $S U(3)_{F}$ antitriplet $\overline{\mathbf{3}}$ (see Fig. 1a), the currents are

$$
\begin{array}{ll}
J_{1}^{\Lambda_{Q}}=\varepsilon_{a b c}\left[u^{a \mathrm{~T}} \mathcal{C} \gamma_{5} d^{b}\right] Q_{v}^{c}, & J_{2}^{\Lambda_{Q}}=\varepsilon_{a b c}\left[u^{a \mathrm{~T}} \mathcal{C} \gamma_{5} \not d^{b}\right] Q_{v}^{c}, \\
J_{1}^{\Xi_{Q}}=\varepsilon_{a b c}\left[q^{a \mathrm{~T}} \mathcal{C} \gamma_{5} s^{b}\right] Q_{v}^{c}, & J_{2}^{\Xi_{Q}}=\varepsilon_{a b c}\left[q^{a \mathrm{~T}} \mathcal{C} \gamma_{5} \not s^{b}\right] Q_{v}^{c},
\end{array}
$$


where $q=u$ or $d$ is one of the isodoublet quark fields. There are also two $S U(3)_{F}$ sextet 6 states (see Fig. 1b), which differ in the total spin of the state. For the $J^{P}=1 / 2^{+}$baryons, the local interpolating currents are

$$
\begin{aligned}
J_{1}^{\Sigma_{Q}}=-\varepsilon_{a b c}\left[q_{1}^{a \mathrm{~T}} \mathcal{C} \gamma_{t \mu} q_{2}^{b}\right] \gamma_{t}^{\mu} \gamma_{5} Q_{v}^{c}, & J_{2}^{\Sigma_{Q}}=-\varepsilon_{a b c}\left[q_{1}^{a \mathrm{~T}} \mathcal{C} \gamma_{t \mu} \phi q_{2}^{b}\right] \gamma_{t}^{\mu} \gamma_{5} Q_{v}^{c}, \\
J_{1}^{\Xi_{Q}^{\prime}}=-\varepsilon_{a b c}\left[q^{a \mathrm{~T}} \mathcal{C} \gamma_{t \mu} s^{b}\right] \gamma_{t}^{\mu} \gamma_{5} Q_{v}^{c}, & J_{2}^{\Xi_{Q}^{\prime}}=-\varepsilon_{a b c}\left[q^{a \mathrm{~T}} \mathcal{C} \gamma_{t \mu} \not s^{b}\right] \gamma_{t}^{\mu} \gamma_{5} Q_{v}^{c}, \\
J_{1}^{\Omega_{Q}}=-\varepsilon_{a b c}\left[s^{a \mathrm{~T}} \mathcal{C} \gamma_{t \mu} s^{b}\right] \gamma_{t}^{\mu} \gamma_{5} Q_{v}^{c}, & J_{2}^{\Omega_{Q}}=-\varepsilon_{a b c}\left[s^{a \mathrm{~T}} \mathcal{C} \gamma_{t \mu} \phi s^{b}\right] \gamma_{t}^{\mu} \gamma_{5} Q_{v}^{c},
\end{aligned}
$$

where $\gamma_{t}^{\mu}=\gamma^{\mu}-\not v^{\mu}$. For the $J^{P}=3 / 2^{+}$baryons, the flavor structure is the same as for the $J^{P}=1 / 2^{+}$ baryons above, but the Dirac structure acting on the heavy-quark field is different. We exemplify such currents by the ones corresponding to the $\Sigma_{Q}^{*}$ baryon:

$$
\begin{aligned}
& J_{1 \mu}^{\Sigma_{Q}^{*}}=\varepsilon_{a b c}\left[q_{1}^{a \mathrm{~T}} \mathcal{C} \gamma_{t}^{\nu} q_{2}^{\prime b}\right]\left(g_{\mu \nu}-\frac{1}{3} \gamma_{t \mu} \gamma_{t \nu}\right) Q_{v}^{c}, \\
& J_{2 \mu}^{\Sigma_{Q}^{*}}=\varepsilon_{a b c}\left[q_{1}^{a \mathrm{~T}} \mathcal{C} \gamma_{t}^{\nu} \not q_{2}^{\prime b}\right]\left(g_{\mu \nu}-\frac{1}{3} \gamma_{t \mu} \gamma_{t \nu}\right) Q_{v}^{c} .
\end{aligned}
$$

These currents satisfy the condition $\gamma_{t}^{\mu} J_{i \mu}^{\Sigma_{Q}^{*}}=0, i=1,2$.

Matrix elements of local operators (2)-(7) define the baryonic couplings $f_{H_{Q}}^{(i)}$ :

$$
\begin{array}{ll}
J^{P}=1 / 2^{+}: & \left\langle 0\left|J_{i}^{H_{Q}}\right| H_{Q}(v)\right\rangle=f_{H_{Q}}^{(i)} u^{H_{Q}}(v), \\
J^{P}=3 / 2^{+}: & \left\langle 0\left|J_{i \mu}^{H_{Q}^{*}}\right| H_{Q}^{*}(v)\right\rangle=\frac{1}{\sqrt{3}} f_{H_{Q}^{*}}^{(i)} u_{\mu}^{H_{Q}^{*}}(v),
\end{array}
$$

where the coefficient in the matrix element $\left\langle 0\left|J_{i \mu}^{H_{Q}^{*}}\right| H_{Q}^{*}(v)\right\rangle$ is chosen such that $f_{H_{Q}}^{(i)}=f_{H_{Q}^{*}}^{(i)}$ in the heavy-quark symmetry limit. The Dirac spinors $u^{H_{Q}}(v)$ of the heavy baryons $H_{Q}$ with the nonrelativistic normalization $\bar{u}^{H_{Q}}(v) u^{H_{Q}}(v)=1$ satisfy the condition $\not u^{H_{Q}}(v)=u^{H_{Q}}(v)$. In the case of the $H_{Q}^{*}$ baryons, the wave function is represented by the Rarita-Schwinger vector spinor $u_{\mu}^{H_{Q}^{*}}(v)$, for which we have the relations $\not u_{\mu}^{H_{Q}^{*}}(v)=u_{\mu}^{H_{Q}^{*}}(v)$ and $v^{\mu} u_{\mu}^{H_{Q}^{*}}(v)=\gamma^{\mu} u_{\mu}^{H_{Q}^{*}}(v)=0 .{ }^{1}$ The sums over their polarizations are [14]

$$
\begin{aligned}
& \sum_{\lambda=1}^{2} u^{H_{Q}(\lambda)}(v) \bar{u}^{H_{Q}(\lambda)}(v)=\frac{1+\not}{2} \equiv P_{+}, \\
& \sum_{\lambda=1}^{4} u_{\mu}^{H_{Q}^{*}(\lambda)}(v) \bar{u}_{\nu}^{H_{Q}^{*}(\lambda)}(v)=P_{+}\left[-g_{\mu \nu}+v_{\mu} v_{\nu}+\frac{1}{3} \gamma_{t \mu} \gamma_{t \nu}\right] .
\end{aligned}
$$

We can easily verify the normalizations of these objects:

$$
\operatorname{Sp} \sum_{\lambda=1}^{2} u^{H_{Q}(\lambda)}(v) \bar{u}^{H_{Q}(\lambda)}(v)=2, \quad-g^{\mu \nu} \mathrm{Sp} \sum_{\lambda=1}^{4} u_{\mu}^{H_{Q}^{*}(\lambda)}(v) \bar{u}_{\nu}^{H_{Q}^{*}(\lambda)}(v)=4,
$$

accounting for the numbers of independent polarization states.

\footnotetext{
${ }^{1} \mathrm{~A}$ method for constructing the spin part of the excited baryons can be found in [14].
} 
The LCDAs of a heavy baryon can be defined through the baryon-to-vacuum matrix elements of suitable nonlocal light-ray operators built from an effective heavy-quark field $Q_{v}^{c}(0)$ and two light-quark fields $q_{i}^{a}, i=1,2$. For the $\Lambda_{Q}$-baryon, the complete set of three-quark light-ray operators with respect to the diquark twist decomposition was recently derived [7]. These currents can be easily adapted to the $\Xi_{Q}$ baryons by replacing one of the light $u$ or $d$ quarks with the $s$ quark and can be written in general form as

$$
\begin{aligned}
& J_{2}\left(t_{1}, t_{2}\right)=\varepsilon_{a b c}\left[q_{1}^{a \mathrm{~T}}\left(t_{1} n\right) \mathcal{C} \gamma_{5} \not h q_{2}^{b}\left(t_{2} n\right)\right] Q_{v}^{c}(0), \\
& J_{3 s}\left(t_{1}, t_{2}\right)=\varepsilon_{a b c}\left[q_{1}^{a \mathrm{~T}}\left(t_{1} n\right) \mathcal{C} \gamma_{5} q_{2}^{b}\left(t_{2} n\right)\right] Q_{v}^{c}(0), \\
& J_{3 \sigma}\left(t_{1}, t_{2}\right)=\frac{i}{2} \varepsilon_{a b c}\left[q_{1}^{a \mathrm{~T}}\left(t_{1} n\right) \mathcal{C} \gamma_{5}(\bar{n} \sigma n) q_{2}^{b}\left(t_{2} n\right)\right] Q_{v}^{c}(0), \\
& J_{4}\left(t_{1}, t_{2}\right)=\varepsilon_{a b c}\left[q_{1}^{a \mathrm{~T}}\left(t_{1} n\right) \mathcal{C} \gamma_{5} \not h q_{2}^{b}\left(t_{2} n\right)\right] Q_{v}^{c}(0),
\end{aligned}
$$

where $(\bar{n} \sigma n)=\bar{n}^{\mu} \sigma_{\mu \nu} n^{\nu}$ and the flavors of the light quarks $q=u, d, s$ are different $\left(q_{1} \neq q_{2}\right)$ (see Fig. 1 for the quark content of real heavy baryons). The subscripts 2, 3, 4 refer to the twist of the diquark operator, and $n^{\mu}$ and $\bar{n}^{\mu}$ are lightlike vectors normalized as $(\bar{n} n)=2$, which we choose such that $v^{\mu}=\left(n^{\mu}+\bar{n}^{\mu}\right) / 2$ and $(n v)=(\bar{n} v)=1$. The matrix elements of operators (13) can be parameterized in accordance with [7]:

$$
\begin{aligned}
& \left\langle 0\left|J_{2}\left(t_{1}, t_{2}\right)\right| H_{Q}(v)\right\rangle=f_{H_{Q}}^{(2)} \Psi_{2}\left(t_{1}, t_{2}\right) u^{H_{Q}}(v), \\
& \left\langle 0\left|J_{3 s}\left(t_{1}, t_{2}\right)\right| H_{Q}(v)\right\rangle=f_{H_{Q}}^{(1)} \Psi_{3 s}\left(t_{1}, t_{2}\right) u^{H_{Q}}(v), \\
& \left\langle 0\left|J_{3 \sigma}\left(t_{1}, t_{2}\right)\right| H_{Q}(v)\right\rangle=f_{H_{Q}}^{(1)} \Psi_{3 \sigma}\left(t_{1}, t_{2}\right) u^{H_{Q}}(v), \\
& \left\langle 0\left|J_{4}\left(t_{1}, t_{2}\right)\right| H_{Q}(v)\right\rangle=f_{H_{Q}}^{(2)} \Psi_{4}\left(t_{1}, t_{2}\right) u^{H_{Q}}(v) .
\end{aligned}
$$

The simplest way to construct the complete set of the three-particle LCDAs of baryons with the diquark spin parity $j^{p}=1^{+}$is to switch off the spin of the heavy quark (we let $\widetilde{Q}_{v}$ denote such a spinless quark) and introduce the LCDAs for the "axial-vector baryon" state $\widetilde{H}_{Q}(v, \eta)$, which in this case is characterized by the polarization vector $\eta^{\mu}$ satisfying the condition $(v \eta)=0$. The LCDA definitions are borrowed from the light-cone analysis of vector mesons [15]. We separate the eight interpolating currents into two groups similar to the chiral-even and chiral-odd LCDAs of a vector meson [15]. The first set is

$$
\begin{aligned}
& J_{2 V}\left(t_{1}, t_{2}\right)=\epsilon_{a b c}\left[q_{1}^{a \mathrm{~T}}\left(t_{1} n\right) \mathcal{C} \not h q_{2}^{b}\left(t_{2} n\right)\right] \widetilde{Q}_{v}^{c}(0), \\
& J_{4 V}\left(t_{1}, t_{2}\right)=\epsilon_{a b c}\left[q_{1}^{a \mathrm{~T}}\left(t_{1} n\right) \mathcal{C} \not h q_{2}^{b}\left(t_{2} n\right)\right] \widetilde{Q}_{v}^{c}(0), \\
& J_{3 V}^{\mu}\left(t_{1}, t_{2}\right)=\epsilon_{a b c}\left[q_{1}^{a \mathrm{~T}}\left(t_{1} n\right) \mathcal{C} \gamma_{\perp}^{\mu} q_{2}^{b}\left(t_{2} n\right)\right] \widetilde{Q}_{v}^{c}(0), \\
& J_{3 A}^{\mu}\left(t_{1}, t_{2}\right)=\epsilon_{a b c} \varepsilon_{\perp}^{\mu \nu}\left[q_{1}^{a \mathrm{~T}}\left(t_{1} n\right) \mathcal{C} \gamma_{\perp \nu} \gamma_{5} q_{2}^{b}\left(t_{2} n\right)\right] \widetilde{Q}_{v}^{c}(0),
\end{aligned}
$$

where $\gamma_{\perp}^{\mu}=\gamma^{\mu}-\left(\not h \bar{n}^{\mu}+\not h n^{\mu}\right) / 2$ and $\varepsilon_{\perp}^{\mu \nu}=(i / 2) \varepsilon^{\mu \nu \rho \sigma} n_{\rho} \bar{n}_{\sigma}$ is the antisymmetric tensor in the plane perpendicular to the light cone (satisfies the condition $\varepsilon_{\perp \mu \nu} \varepsilon_{\perp}^{\nu \mu}=2$ ). The second set is

$$
\begin{aligned}
& J_{2 T}^{\mu}\left(t_{1}, t_{2}\right)=\epsilon_{a b c}\left[q_{1}^{a \mathrm{~T}}\left(t_{1} n\right) \mathcal{C} \gamma_{\perp}^{\mu} \not h q_{2}^{b}\left(t_{2} n\right)\right] \widetilde{Q}_{v}^{c}(0), \\
& J_{4 T}^{\mu}\left(t_{1}, t_{2}\right)=\epsilon_{a b c}\left[q_{1}^{a \mathrm{~T}}\left(t_{1} n\right) \mathcal{C} \gamma_{\perp}^{\mu} \not h q_{2}^{b}\left(t_{2} n\right)\right] \widetilde{Q}_{v}^{c}(0), \\
& J_{3 T}\left(t_{1}, t_{2}\right)=\frac{i}{2} \epsilon_{a b c}\left[q_{1}^{a \mathrm{~T}}\left(t_{1} n\right) \mathcal{C}(\bar{n} \sigma n) q_{2}^{b}\left(t_{2} n\right)\right] \widetilde{Q}_{v}^{c}(0), \\
& J_{3 S}\left(t_{1}, t_{2}\right)=\epsilon_{a b c}\left[q_{1}^{a \mathrm{~T}}\left(t_{1} n\right) \mathcal{C} q_{2}^{b}\left(t_{2} n\right)\right] \widetilde{Q}_{v}^{c}(0) .
\end{aligned}
$$


It is easy to see that linear combinations of currents (15)-(17) and (19)-(21)

$$
\begin{aligned}
& 2 J_{3 V}^{\mu}\left(t_{1}, t_{2}\right)-\bar{v}^{\mu}\left[J_{2 V}\left(t_{1}, t_{2}\right)-J_{4 V}\left(t_{1}, t_{2}\right)\right]=2 \epsilon_{a b c}\left[q_{1}^{a \mathrm{~T}}\left(t_{1} n\right) \mathcal{C} \gamma_{t}^{\mu} q_{2}^{b}\left(t_{2} n\right)\right] \widetilde{Q}_{v}^{c}(0), \\
& J_{2 T}^{\mu}\left(t_{1}, t_{2}\right)+J_{4 T}^{\mu}\left(t_{1}, t_{2}\right)-2 \bar{v}^{\mu} J_{3 T}\left(t_{1}, t_{2}\right)=2 \epsilon_{a b c}\left[q_{1}^{a \mathrm{~T}}\left(t_{1} n\right) \mathcal{C} \gamma_{t}^{\mu} \not q_{2}^{b}\left(t_{2} n\right)\right] \widetilde{Q}_{v}^{c}(0)
\end{aligned}
$$

reduce to the local currents with the nonvanishing matrix elements

$$
\begin{aligned}
& \left\langle 0\left|\epsilon_{a b c}\left[q_{1}^{a}(0) \mathcal{C} \gamma_{t}^{\mu} q_{2}^{b}(0)\right] \widetilde{Q}_{v}^{c}(0)\right| \widetilde{H}_{Q}(v, \eta)\right\rangle=\tilde{\lambda}_{1} \eta^{\mu}, \\
& \left\langle 0\left|\epsilon_{a b c}\left[q_{1}^{a}(0) \mathcal{C} \gamma_{t}^{\mu} \not q_{2}^{b}(0)\right] \widetilde{Q}_{v}^{c}(0)\right| \widetilde{H}_{Q}(v, \eta)\right\rangle=\tilde{\lambda}_{2} \eta^{\mu} .
\end{aligned}
$$

Here, $\bar{v}^{\mu}=\left(n^{\mu}-\bar{n}^{\mu}\right) / 2\left(\right.$ with $\bar{v}^{2}=-1$ and $\left.(v \bar{v})=0\right)$ and $\gamma_{t}^{\mu}=\gamma_{\perp}^{\mu}-\not \bar{p} \bar{v}^{\mu}$. With these definitions, the matrix elements of the nonlocal operators can be determined as

$$
\begin{aligned}
\left\langle 0\left|J_{2 V}\left(t_{1}, t_{2}\right)\right| \widetilde{H}_{Q}(v, \eta)\right\rangle & =\tilde{\lambda}_{1}(n \eta) \Psi_{2 V}\left(t_{1}, t_{2}\right), \\
\left\langle 0\left|J_{3 V}^{\mu}\left(t_{1}, t_{2}\right)\right| \widetilde{H}_{Q}(v, \eta)\right\rangle & =\tilde{\lambda}_{1} \eta_{\perp}^{\mu} \Psi_{3 V}\left(t_{1}, t_{2}\right), \\
\left\langle 0\left|J_{4 V}\left(t_{1}, t_{2}\right)\right| \widetilde{H}_{Q}(v, \eta)\right\rangle & =-\tilde{\lambda}_{1}(\bar{n} \eta) \Psi_{4 V}\left(t_{1}, t_{2}\right), \\
\left\langle 0\left|J_{3 A}^{\mu}\left(t_{1}, t_{2}\right)\right| \widetilde{H}_{Q}(v, \eta)\right\rangle & =\tilde{\lambda}_{1} \eta_{\perp}^{\mu} \Psi_{3 A}\left(t_{1}, t_{2}\right), \\
\left\langle 0\left|J_{2 T}^{\mu}\left(t_{1}, t_{2}\right)\right| \widetilde{H}_{Q}(v, \eta)\right\rangle & =\tilde{\lambda}_{2} \eta_{\perp}^{\mu} \Psi_{2 T}\left(t_{1}, t_{2}\right), \\
\left\langle 0\left|J_{3 T}\left(t_{1}, t_{2}\right)\right| \widetilde{H}_{Q}(v, \eta)\right\rangle & =\tilde{\lambda}_{2}(\bar{v} \eta) \Psi_{3 T}\left(t_{1}, t_{2}\right), \\
\left\langle 0\left|J_{4 T}^{\mu}\left(t_{1}, t_{2}\right)\right| \widetilde{H}_{Q}(v, \eta)\right\rangle & =\tilde{\lambda}_{2} \eta_{\perp}^{\mu} \Psi_{4 T}\left(t_{1}, t_{2}\right), \\
\left\langle 0\left|J_{3 S}\left(t_{1}, t_{2}\right)\right| \widetilde{H}_{Q}(v, \eta)\right\rangle & =\tilde{\lambda}_{2}(\bar{v} \eta) \Psi_{3 S}\left(t_{1}, t_{2}\right),
\end{aligned}
$$

where $\eta_{\perp}^{\mu}=\eta^{\mu}+\bar{v}^{\mu}(\bar{v} \eta)$. In the $S U(3)_{F}$-symmetry limit, the LCDAs $\Psi_{i V}\left(t_{1}, t_{2}\right)$ and $\Psi_{i T}\left(t_{1}, t_{2}\right)$ are symmetric under the exchange $t_{1} \leftrightarrow t_{2}$ and normalized as $\Psi_{i V}(0,0)=\Psi_{i T}(0,0)=1$, and $\Psi_{3 S}\left(t_{1}, t_{2}\right)$ and $\Psi_{3 A}\left(t_{1}, t_{2}\right)$ are antisymmetric and hence satisfy the condition $\Psi_{3 A}(0,0)=\Psi_{3 S}(0,0)=0$. The breaking of the $S U(3)_{F}$-symmetry results in the violation of the LCDA symmetry properties.

Passing to the real heavy-quark field $Q_{v}^{c}(0)$ in nonlocal currents (15)-(22) is equivalent to replacing $\widetilde{Q}_{v}^{c}(0) \rightarrow \Gamma^{\prime} Q_{v}^{c}(0)$, where the Dirac matrix $\Gamma^{\prime}$ gives the correct quantum numbers of the specific baryon. This also improves the right-hand side of definitions (27)-(34), but the set of the LCDAs remains the same (see [8] for the details).

\section{Scale dependence of matrix elements}

A nonrelativistic constituent quark picture of the $H_{Q}$ baryon suggests that $f_{H_{Q}}^{(2)} \simeq f_{H_{Q}}^{(1)}$ at low scales of the order of $1 \mathrm{GeV}$, and this expectation is supported by numerous QCD sum-rule calculations [10], [11], [16], [17]. In fact, the difference between the two couplings is only obtained at the level of NLO perturbative corrections to the sum rules [11], [17] and is numerically small.

The scale dependence of the couplings is given by

$$
f_{H_{Q}}^{(i)}(\mu)=f_{H_{Q}}^{(i)}\left(\mu_{0}\right)\left(\frac{\alpha_{s}(\mu)}{\alpha_{s}\left(\mu_{0}\right)}\right)^{\gamma_{1}^{(i)} / \beta_{0}}\left[1-\frac{\alpha_{s}\left(\mu_{0}\right)-\alpha_{s}(\mu)}{4 \pi} \frac{\gamma_{1}^{(i)}}{\beta_{0}}\left(\frac{\gamma_{2}^{(i)}}{\gamma_{1}^{(i)}}-\frac{\beta_{1}}{\beta_{0}}\right)\right]
$$


where the anomalous dimensions of the local interpolating operators

$$
\frac{d \log f_{H_{Q}}^{(i)}(\mu)}{d \log \mu} \equiv-\gamma^{(i)}=-\sum_{k} \gamma_{k}^{(i)} a^{k}(\mu), \quad a(\mu) \equiv \frac{\alpha_{s}^{\overline{\mathrm{MS}}}(\mu)}{4 \pi}
$$

are known to the NLO [17] and the NLO expansion of the $\beta$-function

$$
\frac{d a(\mu)}{d \log \mu}=-\beta_{0} a^{2}(\mu)-\beta_{1} a^{3}(\mu)+\cdots
$$

is used with the coefficients $\beta_{0}=2\left(11-2 n_{f} / 3\right)$ and $\beta_{1}=4\left(51-19 n_{f} / 3\right)$.

For the numerical values of the couplings for the bottom baryons, we quote the result of the NLO QCD sum-rule analysis in [7], [11]:

$$
f_{\Lambda_{b}}^{(1)}(1 \mathrm{GeV}) \simeq f_{\Lambda_{b}}^{(2)}(1 \mathrm{GeV}) \simeq(0.030 \pm 0.005) \mathrm{GeV}^{3}
$$

at the renormalization scale $\mu=1 \mathrm{GeV}$. We note that these couplings differ at an arbitrary scale because the corresponding operators have different anomalous dimensions.

The LCDAs introduced in Sec. 2 depend on the scale. To determine this dependence, it is convenient to pass to the momentum representation of LCDAs using the Fourier transformation,

$$
\begin{aligned}
\Psi_{k}\left(t_{1}, t_{2}\right) & =\int_{0}^{\infty} d \omega_{1} \int_{0}^{\infty} d \omega_{2} e^{-i\left(t_{1} \omega_{1}+t_{2} \omega_{2}\right)} \psi_{k}\left(\omega_{1}, \omega_{2}\right)= \\
& =\int_{0}^{\infty} \omega d \omega \int_{0}^{1} d u e^{-i \omega\left(t_{1} u+t_{2} \bar{u}\right)} \widetilde{\psi}_{k}(\omega, u),
\end{aligned}
$$

and hence $\widetilde{\psi}_{k}(\omega, u)=\psi_{k}(u \omega, \bar{u} \omega)$ with $\bar{u}=1-u$. In the first representation, $\omega_{1}$ and $\omega_{2}$ are the energies of the light quarks, and in the second, $\omega=\omega_{1}+\omega_{2}$ is the total energy carried by light quarks (in the heavy-quark rest frame) while the dimensionless variable $u$ corresponds to the energy fraction carried by the quark called $q_{1}$.

The leading-order evolution equation for the leading-twist LCDA $\psi_{2}\left(\omega_{1}, \omega_{2} ; \mu\right)$ can be derived by the usual procedure [7], identifying the ultraviolet singularities of one-gluon-exchange diagrams shown in Fig 2 . The result can be expressed in terms of the two-particle kernels familiar from the evolution equations for the $B$ - and $\pi$-meson LCDAs [7]:

$$
\begin{aligned}
\mu \frac{d}{d \mu} \psi_{2}\left(\omega_{1}, \omega_{2} ; \mu\right)= & -\frac{\alpha_{s}(\mu)}{2 \pi}\left(1+\frac{1}{N_{c}}\right)\left\{\int_{0}^{\infty} d \omega_{1}^{\prime} \gamma^{\mathrm{LN}}\left(\omega_{1}^{\prime}, \omega_{1} ; \mu\right) \psi_{2}\left(\omega_{1}^{\prime}, \omega_{2} ; \mu\right)+\right. \\
& +\int_{0}^{\infty} d \omega_{2}^{\prime} \gamma^{\mathrm{LN}}\left(\omega_{2}^{\prime}, \omega_{2} ; \mu\right) \psi_{2}\left(\omega_{1}, \omega_{2}^{\prime} ; \mu\right)- \\
& \left.-\int_{0}^{1} d v V(u, v) \psi_{2}(v \omega, \bar{v} \omega ; \mu)+\frac{3}{2} \psi_{2}\left(\omega_{1}, \omega_{2} ; \mu\right)\right\}
\end{aligned}
$$

where the last term in the braces, $3 \psi_{2}\left(\omega_{1}, \omega_{2} ; \mu\right) / 2$, is a result of subtracting the one-loop renormalization of the coupling $f_{H_{Q}}^{(2)}$.

The first two convolution integrals in Eq. (40) are associated with the heavy-light dynamics: each of them involves just one of the light quarks. Indeed, the kernel $\gamma^{\mathrm{LN}}\left(\omega^{\prime}, \omega ; \mu\right)$ coincides with the one controlling the evolution of the $B$-meson distribution amplitude, the Lange-Neubert anomalous dimension [18]. In 

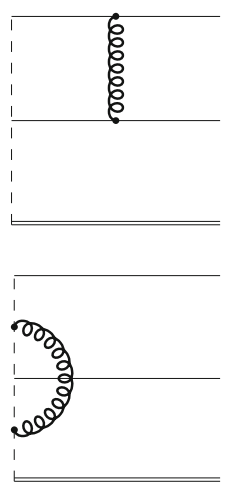
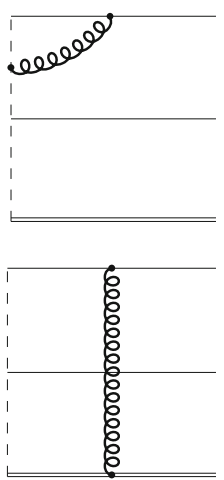
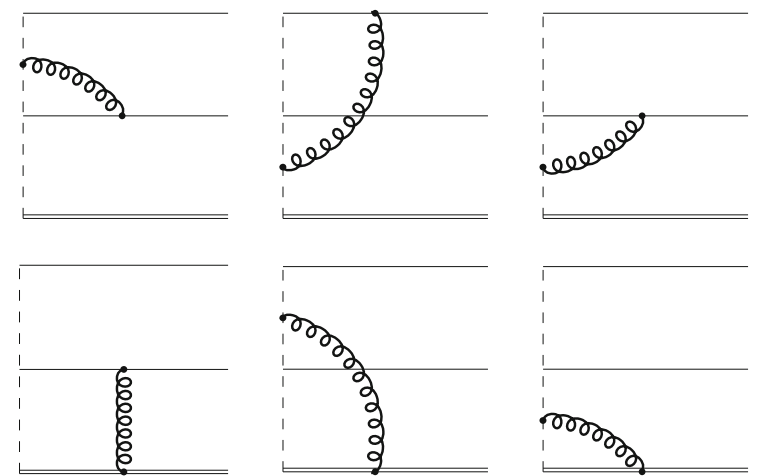

Fig. 2. The total set of one-gluon-exchange diagrams for calculating the scale dependence of LCDAs of the heavy baryon: normal and bold solid lines respectively correspond to light and heavy quarks, and dashed and curly lines describe the Wilson links and exchanged gluons.

turn, the last convolution integral describes the interaction between the light quarks, where $V(u, v)$ is the well-known Efremov-Radyushkin-Brodsky-Lepage kernel [19].

For small evolution ranges, $\log \left(\mu / \mu_{0}\right) \lesssim 1$, it suffices to interpret the derivative in the left-hand side of (40) as a finite difference $\left[\psi_{2}\left(\omega_{1}, \omega_{2} ; \mu\right)-\psi_{2}\left(\omega_{1}, \omega_{2} ; \mu_{0}\right)\right] / \log \left(\mu / \mu_{0}\right)$ and substitute the initial condition $\psi_{2}\left(\omega_{1}, \omega_{2} ; \mu_{0}\right)$ for $\psi_{2}\left(\omega_{1}, \omega_{2} ; \mu\right)$ in the right-hand side. Obviously, this corresponds to taking only the one-loop renormalization into account, neglecting the resummation of potentially large logarithms. As demonstrated in [7], this single-evolution-step (one-loop) approximation is quite good in practice, i.e., for $\mu_{0}=1 \mathrm{GeV}$ and $\mu \simeq m_{b} / 2$, where $m_{b}$ is the $b$-quark mass. To go beyond the one-loop approximation, one possibility is to integrate evolution equation (40) numerically. The other (semianalytic) approach was suggested in [7]; it has an advantage that it allows understanding the structure of the solution qualitatively.

\section{The QCD sum rules}

Models for the heavy-baryon LCDAs can be obtained by applying the QCD sum rules to correlation functions constructed from the nonlocal light-ray operator $\mathcal{O}^{\Gamma}\left(t_{1}, t_{2}\right)$ and a suitable local current $\bar{J}^{\Gamma^{\prime}}(x)$, as shown in Fig. 3. As a suitable local current of heavy baryons, we can take

$$
\bar{J}^{\Gamma^{\prime}}(x)=A \bar{J}_{1}^{H_{Q}}+B \bar{J}_{2}^{H_{Q}}=\varepsilon_{a b c}\left[\bar{q}_{2}^{a}(x)(A+B \not b) \Gamma^{\prime} \mathcal{C}^{\mathrm{T}} \bar{q}_{1}^{b \mathrm{~T}}(x)\right] \bar{Q}_{v}^{c}(x),
$$

where $\mathcal{C}^{\mathrm{T}}$ is the transposed matrix $\mathcal{C}$. Varying the coefficients $A$ and $B$, we can estimate the uncertainty arising from the choice of the local current. The currents $\bar{J}_{1}^{H_{Q}}(x)$ and $\bar{J}_{2}^{H_{Q}}(x)$ were used in [11] to write the diagonal, nondiagonal, and constituent-type sum rules. For the baryons with the diquark spin parity $j^{p}=0^{+}$, we should take $\Gamma^{\prime}=\gamma_{5}$; for the $j^{p}=1^{+}$diquark, there are two possibilities $\Gamma^{\prime}=\gamma_{\|}$or $\Gamma^{\prime}=\gamma_{\perp}$. The current $\bar{J}_{\Lambda}(x)$ chosen in [7] for the $\Lambda_{b}$ baryon with the spin parity $j^{p}=0^{+}$of the diquark is just current (41) with $A=B=1 / 2$, which picks up the contributions of both even and odd dimensions, i.e., $\bar{J}_{2}^{\Lambda_{b}}(x)$ produces the perturbative theory and quartic condensate contributions, while $\bar{J}_{1}^{\Lambda_{b}}(x)$ results in the nonlocal quark condensates.

The procedure for constructing the QCD sum rules is well known and leads to the general form

$$
f_{k}\left(A f_{H_{Q}}^{(1)}+B f_{H_{Q}}^{(2)}\right) \widetilde{\psi}_{k}^{\mathrm{SR}}\left(t_{1}, t_{2}\right) e^{-\bar{\Lambda} / \tau}=\mathcal{B}\left[\Pi_{k}\right]\left(t_{1}, t_{2} ; \tau, s_{0}\right),
$$

where $f_{k}=f_{H_{Q}}^{(2)}$ for the even twists and $f_{k}=f_{H_{Q}}^{(1)}$ for the twist-3 distribution amplitudes. The effective baryon mass is introduced as the difference $\bar{\Lambda}=m_{H_{Q}}-m_{Q}$, where $m_{Q}$ is the mass of the heavy quark, 


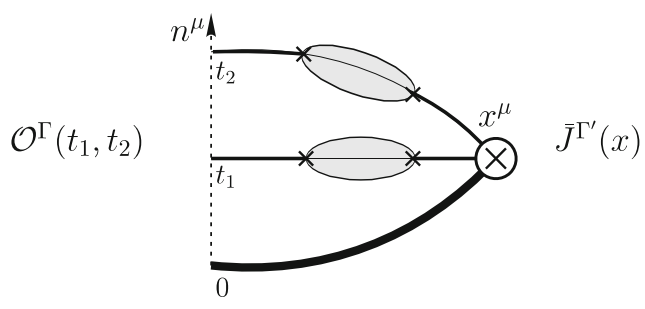

Fig. 3. The correlation function constructed from the nonlocal light-ray operator $\mathcal{O}^{\Gamma}\left(t_{1}, t_{2}\right)$ and a local current $\bar{J}^{\Gamma^{\prime}}(x)$ : the bold solid line corresponds to the heavy-quark propagator, and the solid lines with the blob insertion are the propagators of the light quarks, modified by contributions of the nonlocal quark condensates.

$\tau$ is the Borel parameter, and $s_{0}$ is the continuum threshold. The right-hand side of Eq. (42) is the Borel-transformed continuum-subtracted invariant function determined in terms of the correlation function $\Pi_{k}\left(t_{1}, t_{2} ; \tau\right)$.

For practical applications, we must know LCDAs in the momentum space. The Fourier transform of LCDAs is defined in Eq. (39), and for the scalar correlation function, we take the definition

$$
\Pi_{k}(\omega, u ; \tau)=\int_{-\infty}^{\infty} \frac{d t_{1}}{2 \pi} \int_{-\infty}^{\infty} \frac{d t_{2}}{2 \pi} e^{i \omega\left(u t_{1}+\bar{u}_{2}\right)} \Pi_{k}\left(t_{1}, t_{2} ; \tau\right)
$$

In the momentum space, sum rules (42) can be written in the form

$$
f_{k}\left(A f_{H_{Q}}^{(1)}+B f_{H_{Q}}^{(2)}\right) \widetilde{\psi}_{k}^{\mathrm{SR}}(\omega, u) e^{-\bar{\Lambda} / \tau}=\mathcal{B}\left[\Pi_{k}\right]\left(\omega, u ; \tau, s_{0}\right)
$$

Taking only the leading-order perturbative contribution to the sum rules into account, we obtain

$$
\begin{aligned}
\widetilde{\psi}_{2}(\omega, u)= & \frac{30 \tau^{4}}{\mathcal{N}}\left[\widehat{\omega}^{2} u \bar{u}+\frac{A}{B} \widehat{\omega}\left(\widehat{m}_{2} u+\widehat{m}_{1} \bar{u}\right)\right] E_{1}\left(2 \hat{s}_{\omega}\right) e^{-\widehat{\omega}}, \\
\widetilde{\psi}_{4}(\omega, u)= & \frac{30 \tau^{4}}{\mathcal{N}}\left[E_{3}\left(2 \hat{s}_{\omega}\right)+\frac{A}{B}\left(\widehat{m}_{1}+\widehat{m}_{2}\right) E_{2}\left(2 \hat{s}_{\omega}\right)\right] e^{-\widehat{\omega}}, \\
\widetilde{\psi}_{3 s}(\omega, u)= & \frac{15 \tau^{4}}{\mathcal{N}}\left\{\left[\widehat{\omega}+\frac{B}{A}\left(\widehat{m}_{1}+\widehat{m}_{2}\right)\right] E_{2}\left(2 \hat{s}_{\omega}\right)+\right. \\
& \left.+\frac{B}{A} \widehat{\omega}\left(\widehat{m}_{2} u+\widehat{m}_{1} \bar{u}\right) E_{1}\left(2 \hat{s}_{\omega}\right)\right\} e^{-\widehat{\omega}}, \\
\widetilde{\psi}_{3 \sigma}(\omega, u)= & \frac{15 \tau^{4}}{\mathcal{N}}\left\{\left[\widehat{\omega}(u-\bar{u})+\frac{B}{A}\left(\widehat{m}_{1}-\widehat{m}_{2}\right)\right] E_{2}\left(2 \hat{s}_{\omega}\right)+\right. \\
& \left.+\frac{B}{A} \widehat{\omega}\left(\widehat{m}_{2} u-\widehat{m}_{1} \bar{u}\right) E_{1}\left(2 \hat{s}_{\omega}\right)\right\} e^{-\widehat{\omega}},
\end{aligned}
$$

where $s_{\omega}=s_{0}-\omega / 2, \widehat{\omega}=\omega /(2 \tau), \hat{s}_{\omega}=s_{\omega} /(2 \tau)$, and $\widehat{m}_{1,2}=m_{1,2} /(2 \tau)$. The normalization integral $\mathcal{N}$ is introduced via the baryonic coupling:

$$
\left|f_{H_{Q}}^{(i)}\right|^{2} e^{-\bar{\Lambda} / \tau}=\frac{1}{20 \pi^{4}} \int_{0}^{s_{0}} d s s^{5} e^{-s / \tau} \equiv \frac{\mathcal{N}}{20 \pi^{4}}
$$


For convenience in writing Eqs. (45)-(48), we introduced the function

$$
E_{a}(x)=\frac{1}{\Gamma(a+1)} \int_{0}^{x} d t t^{a} e^{-t}=1-\frac{\Gamma(a+1, x)}{\Gamma(a+1)}
$$

where $\Gamma(a+1, x)=\int_{x}^{\infty} d t t^{a} e^{-t}$ is the incomplete $\Gamma$-function. For integer values of the parameter $(a=N)$, this function reduces to the well-known form

$$
E_{N}(x)=1-e^{-x} \sum_{n=0}^{N} \frac{x^{n}}{n !} .
$$

For practical numerical estimates, it is more convenient to integrate by parts,

$$
E_{a}(x)=E_{a+1}(x)+\frac{x^{a+1} e^{-x}}{\Gamma(a+2)}
$$

to convert a negative value of the parameter $a$ in $E_{a}(x)$ into a positive value, as was done in [7].

To evaluate the nonperturbative contributions to the sum rules, we can use the nonlocal quark condensates used for the $B$-meson case in [20], [21]. In the analysis here, we use the general parameterization for the nonlocal quark condensate [22], [23]

$$
\langle\bar{q}(x) q(0)\rangle=\langle\bar{q} q\rangle \int_{0}^{\infty} d \nu e^{\nu x^{2} / 4} f(\nu)
$$

where $\langle\bar{q} q\rangle$ is the local quark condensate and $f(\nu)$ is the model function [21], [24]

$$
f(\nu)=\frac{\lambda^{a-2}}{\Gamma(a-2)} \nu^{1-a} e^{-\lambda / \nu}, \quad a-3=\frac{4 \lambda}{m_{0}^{2}} .
$$

The parameters $\lambda$ and $m_{0}^{2}$ in the model function $f(\nu)$ have the meanings of the correlation length and the ratio of the local mixed quark-gluon condensate to the quark condensate. The nonlocal quark and gluon condensates of higher dimensions were comprehensively analyzed in [25].

Taking the nonlocal quark condensates into account, we write the sum rules as

$$
\begin{aligned}
f_{H_{Q}}^{(2)}\left(A f_{H_{Q}}^{(1)}+B f_{H_{Q}}^{(2)}\right) \widetilde{\psi}_{2}^{\mathrm{SR}}(\omega, u) e^{-\bar{\Lambda} / \tau}= \\
=\frac{3 \tau^{4}}{2 \pi^{4}}\left[B \widehat{\omega}^{2} u \bar{u}\left(\widehat{\omega}^{2} u \bar{u}+\widehat{m}_{1} \widehat{m}_{2}\right)+A \widehat{\omega}\left(\widehat{m}_{2} u+\widehat{m}_{1} \bar{u}\right)\right] E_{1}\left(2 \hat{s}_{\omega}\right) e^{-\widehat{\omega}}- \\
\quad-\frac{\left\langle\bar{q}_{1} q_{1}\right\rangle \tau^{3}}{\pi^{2}}\left[A \widehat{\omega} \bar{u}+B \widehat{m}_{2}\right] f(2 \tau \omega u) E_{2-a}\left(2 \hat{s}_{\kappa}\right) e^{-\widehat{\omega}}- \\
\quad-\frac{\left\langle\bar{q}_{2} q_{2}\right\rangle \tau^{3}}{\pi^{2}}\left[A \widehat{\omega} u+B \widehat{m}_{1}\right] f(2 \tau \omega \bar{u}) E_{2-a}\left(2 \hat{s}_{\bar{\kappa}}\right) e^{-\widehat{\omega}}+ \\
\quad+\frac{2 B}{3}\left\langle\bar{q}_{1} q_{1}\right\rangle\left\langle\bar{q}_{2} q_{2}\right\rangle \tau^{2} f(2 \tau \omega u) f(2 \tau \omega \bar{u}) E_{3-2 a}\left(2 \hat{s}_{\kappa \bar{\kappa}}\right) e^{-\widehat{\omega}},
\end{aligned}
$$




$$
\begin{aligned}
& f_{H_{Q}}^{(2)}\left(A f_{H_{Q}}^{(1)}+B f_{H_{Q}}^{(2)}\right) \widetilde{\psi}_{4}^{\mathrm{SR}}(\omega, u) e^{-\bar{\Lambda} / \tau}= \\
& =\frac{3 \tau^{4}}{2 \pi^{4}}\left[B E_{3}\left(2 \hat{s}_{\omega}\right)+A\left(\widehat{m}_{1}+\widehat{m}_{2}\right) E_{2}\left(2 \hat{s}_{\omega}\right)\right] e^{-\widehat{\omega}}- \\
& -\frac{\left\langle\bar{q}_{1} q_{1}\right\rangle \tau^{3}}{\pi^{2}}\left[A E_{3-a}\left(2 \hat{s}_{\kappa}\right)+B \widehat{m}_{2} E_{2-a}\left(2 \hat{s}_{\kappa}\right)\right] f(2 \tau \omega u) e^{-\widehat{\omega}}- \\
& -\frac{\left\langle\bar{q}_{2} q_{2}\right\rangle \tau^{3}}{\pi^{2}}\left[A E_{3-a}\left(2 \hat{s}_{\bar{\kappa}}\right)+B \widehat{m}_{1} E_{2-a}\left(2 \hat{s}_{\bar{\kappa}}\right)\right] f(2 \tau \omega \bar{u}) e^{-\widehat{\omega}}+ \\
& +\frac{2 B}{3}\left\langle\bar{q}_{1} q_{1}\right\rangle\left\langle\bar{q}_{2} q_{2}\right\rangle \tau^{2} f(2 \tau \omega u) f(2 \tau \omega \bar{u}) E_{3-2 a}\left(2 \hat{s}_{\kappa \bar{\kappa}}\right) e^{-\widehat{\omega}}, \\
& f_{H_{Q}}^{(1)}\left(A f_{H_{Q}}^{(1)}+B f_{H_{Q}}^{(2)}\right) \widetilde{\psi}_{3 s}^{\mathrm{SR}}(\omega, u) e^{-\bar{\Lambda} / \tau}= \\
& =\frac{3 \tau^{4}}{4 \pi^{4}}\left\{\left[A \widehat{\omega}+B\left(\widehat{m}_{1}+\widehat{m}_{2}\right)\right] E_{2}\left(2 \hat{s}_{\omega}\right)+B \widehat{\omega}\left(\widehat{m}_{2} u+\widehat{m}_{1} \bar{u}\right) E_{1}\left(2 \hat{s}_{\omega}\right)\right\} e^{-\widehat{\omega}}- \\
& -\frac{\left\langle\bar{q}_{1} q_{1}\right\rangle \tau^{3}}{2 \pi^{2}}\left[B E_{3-a}\left(2 \hat{s}_{\kappa}\right)+\left(B \widehat{\omega} \bar{u}+2 A \widehat{m}_{2}\right) E_{2-a}\left(2 \hat{s}_{\kappa}\right)\right] f(2 \tau \omega u) e^{-\widehat{\omega}}- \\
& -\frac{\left\langle\bar{q}_{2} q_{2}\right\rangle \tau^{3}}{2 \pi^{2}}\left[B E_{3-a}\left(2 \hat{s}_{\bar{\kappa}}\right)+\left(B \widehat{\omega} u+2 A \widehat{m}_{1}\right) E_{2-a}\left(2 \hat{s}_{\bar{\kappa}}\right)\right] f(2 \tau \omega \bar{u}) e^{-\widehat{\omega}}+ \\
& +\frac{2 A}{3}\left\langle\bar{q}_{1} q_{1}\right\rangle\left\langle\bar{q}_{2} q_{2}\right\rangle \tau^{2} f(2 \tau \omega u) f(2 \tau \omega \bar{u}) E_{3-2 a}\left(2 \hat{s}_{\kappa \bar{\kappa}}\right) e^{-\widehat{\omega}}, \\
& f_{H_{Q}}^{(1)}\left(A f_{H_{Q}}^{(1)}+B f_{H_{Q}}^{(2)}\right) \widetilde{\psi}_{3 \sigma}^{\mathrm{SR}}(\omega, u) e^{-\bar{\Lambda} / \tau}= \\
& =\frac{3 \tau^{4}}{4 \pi^{4}}\left\{\left[A \widehat{\omega}(u-\bar{u})+B\left(\widehat{m}_{1}-\widehat{m}_{2}\right)\right] E_{2}\left(2 \hat{s}_{\omega}\right)+B \widehat{\omega}\left(\widehat{m}_{2} u-\widehat{m}_{1} \bar{u}\right) E_{1}\left(2 \hat{s}_{\omega}\right)\right\} e^{-\widehat{\omega}}- \\
& -\frac{B\left\langle\bar{q}_{1} q_{1}\right\rangle \tau^{3}}{2 \pi^{2}}\left[E_{3-a}\left(2 \hat{s}_{\kappa}\right)-\widehat{\omega} \bar{u} E_{2-a}\left(2 \hat{s}_{\kappa}\right)\right] f(2 \tau \omega u) e^{-\widehat{\omega}}+ \\
& +\frac{B\left\langle\bar{q}_{2} q_{2}\right\rangle \tau^{3}}{2 \pi^{2}}\left[E_{3-a}\left(2 \hat{s}_{\bar{\kappa}}\right)-\widehat{\omega} u E_{2-a}\left(2 \hat{s}_{\bar{\kappa}}\right)\right] f(2 \tau \omega \bar{u}) e^{-\widehat{\omega}},
\end{aligned}
$$

where $\hat{s}_{\kappa}=\hat{s}_{\omega}-\kappa / 2, \hat{s}_{\bar{\kappa}}=\hat{s}_{\omega}-\bar{\kappa} / 2, \hat{s}_{\kappa \bar{\kappa}}=\hat{s}_{\omega}-\kappa / 2-\bar{\kappa} / 2, \kappa=\lambda /(2 u \omega \tau)$, and $\bar{\kappa}=\lambda /(2 \bar{u} \omega \tau)$. The QCD sum rules for twist-2 LCDA (55) coincide with those in [7] in the limit of massless light quarks $\widehat{m}_{1}=\widehat{m}_{2}=0$ when $A=B=1 / 2$. The impact of the quark-hadron duality on the double condensate terms in Eqs. (55)(57) is the appearance of the function $E_{3-2 a}\left(2 \hat{s}_{\kappa \bar{\kappa}}\right)$, which in the local limit becomes unity in agreement with the original result [7].

The QCD sum rules for heavy baryons containing the diquark with the spin parity $j^{p}=1^{+}$can be constructed similarly. The corresponding equations will be presented in [8].

\section{Numerical analysis}

To perform a numerical analysis, it is necessary to specify the required input parameters. The values of the effective baryon masses $\bar{\Lambda}=m_{H_{b}}-m_{b}$ in the HQET for $m_{b}=4.8 \mathrm{GeV}$ are presented in Table 1 , where experimental measurements [26] and theoretical predictions (based on the HQET [27] and lattice QCD [28] for the masses (in units of $\mathrm{MeV}$ ) of the ground-state bottom baryons are also shown. A comparative analysis of predictions for the heavy baryon masses can be found in [27], [29]. The continuum threshold values $s_{0}$ (the last column in Table 1) that we use agree with those derived for the baryon mass evaluation to the order $1 / m_{b}$ in the HQET. The other input parameters are presented in Table 2 (see [30] and the references 
therein for a discussion of these parameters). We note that the shape function $f(\nu)$ in nonlocal quark condensate (53) is assumed to be independent of the flavor for all light quarks.

Table 1

\begin{tabular}{|ccccccc|}
\hline Baryon & $J^{P}$ & Experiment $[26]$ & HQET $[27]$ & Lattice QCD $[28]$ & $\bar{\Lambda}$ & $s_{0}$ \\
\hline$\Lambda_{b}$ & $1 / 2^{+}$ & $5620.0 \pm 1.6$ & $5637_{-56}^{+68}$ & $5641 \pm 21_{-33}^{+15}$ & 0.8 & 1.2 \\
$\Sigma_{b}^{+}$ & $1 / 2^{+}$ & $5807.8 \pm 2.7$ & $5809_{-76}^{+82}$ & $5795 \pm 16_{-26}^{+17}$ & 1.0 & 1.3 \\
$\Sigma_{b}^{-}$ & $1 / 2^{+}$ & $5815.2 \pm 2.0$ & $5809_{-76}^{+82}$ & $5795 \pm 16_{-26}^{+17}$ & 1.0 & 1.3 \\
$\Sigma_{b}^{*+}$ & $3 / 2^{+}$ & $5829.0 \pm 3.4$ & $5835_{-77}^{+82}$ & $5842 \pm 26_{-18}^{+20}$ & 1.0 & 1.3 \\
$\Sigma_{b}^{*-}$ & $3 / 2^{+}$ & $5836.4 \pm 2.8$ & $5835_{-77}^{+82}$ & $5842 \pm 26_{-18}^{+20}$ & 1.0 & 1.3 \\
$\Xi_{b}^{-}$ & $1 / 2^{+}$ & $5790.5 \pm 2.7$ & $5780_{-68}^{+73}$ & $5781 \pm 17_{-16}^{+17}$ & 1.0 & 1.3 \\
$\Xi_{b}^{\prime}$ & $1 / 2^{+}$ & & $5903_{-79}^{+81}$ & $5903 \pm 12_{-19}^{+18}$ & 1.1 & 1.4 \\
$\Xi_{b}^{\prime *}$ & $3 / 2^{+}$ & & $5903_{-79}^{+81}$ & $5950 \pm 21_{-21}^{+19}$ & 1.1 & 1.4 \\
$\Omega_{b}^{-}$ & $1 / 2^{+}$ & $6071 \pm 40$ & $6036 \pm 81$ & $6006 \pm 10_{-19}^{+20}$ & 1.3 & 1.5 \\
$\Omega_{b}^{*}$ & $3 / 2^{+}$ & & $6063_{-82}^{+83}$ & $6044 \pm 18_{-21}^{+20}$ & 1.3 & 1.5 \\
\hline
\end{tabular}

Table 2

\begin{tabular}{|lc|lc|}
\hline$\tau$ & $(0.6 \pm 0.2) \mathrm{GeV}$ & $m_{s}(1 \mathrm{GeV})$ & $(128 \pm 21) \mathrm{MeV}$ \\
$\langle\bar{q} q\rangle(1 \mathrm{GeV})$ & $-\left(242_{-19}^{+28}\right) \mathrm{MeV}^{3}$ & $\langle\bar{s} s\rangle /\langle\bar{q} q\rangle$ & $0.8 \pm 0.2$ \\
$m_{0}^{2}$ & $(0.8 \pm 0.2) \mathrm{GeV}^{2}$ & $\lambda$ & $0.16 \mathrm{GeV}^{2}$ \\
\hline
\end{tabular}

Using these QCD sum rules, we can calculate LCDA moments, certain integrals of the fraction of the momentum carried by the quarks. We define such moments as

$$
\langle f(\omega, u)\rangle_{t}^{H_{Q}} \equiv \int_{0}^{2 s_{0}} \omega d \omega \int_{0}^{1} d u f(\omega, u) \widetilde{\psi}_{t}^{\mathrm{SR}}(\omega, u)
$$

where $t=2,3 s, 3 \sigma, 4$. The normalization of the leading twist $(t=2)$ LCDA is fixed by the condition

$$
\int_{0}^{2 s_{0}} \omega d \omega \int_{0}^{1} d u \widetilde{\psi}_{2}^{\mathrm{SR}}(\omega, u) \equiv 1
$$

For the twist-2 LCDA, it is convenient to expand in terms of the Gegenbauer polynomials $C_{n}^{3 / 2}(2 u-1)$, which (in the massless limit) are orthogonal with respect to the LCDA asymptotic behavior $\sim u(1-u)$ (see (55)). The errors correspond to the variation of the parameters $A$ and $B$ in the ranges $0 \leq A, B \leq 1$ under the condition $A+B=1$, and the central values of the other input parameters are taken from Table 2. As a verification, we reproduced the numerical values for the moments [7] corresponding to the leading twist LCDA of the $\Lambda_{b}$ baryon. All the moments of the $\Lambda_{b}$ baryon calculated with respect to oddorder Gegenbauer polynomials are equal to zero because this function is symmetric under the interchange $u \leftrightarrow 1-u$. Because of the nonzero $s$-quark mass and the difference between the strange $\langle\bar{s} s\rangle$ and nonstrange $\langle\bar{q} q\rangle$ local condensates, this is no longer true for the $\Xi_{b}$ baryon if the $S U(3)_{F}$-breaking corrections are taken into account. It can be easily seen from Table 3 that these corrections typically yield an effect $\sim 10 \%$.

We also use normalization condition (60) for the LCDAs of twist-3 $\widetilde{\psi}_{3 s}^{\mathrm{SR}}(\omega, u)$ and twist- $4 \widetilde{\psi}_{4}^{\mathrm{SR}}(\omega, u)$. Integral (60) with the LCDA $\widetilde{\psi}_{3 \sigma}^{\mathrm{SR}}(\omega, u)$ turns out to be zero in the $S U(3)_{F}$-symmetry limit because this function is antisymmetric under the interchange $u \leftrightarrow 1-u$. To avoid this problem, we use the normalization condition for $\widetilde{\psi}_{3 \sigma}^{\mathrm{SR}}(\omega, u)$

$$
\int_{0}^{2 s_{0}} \omega d \omega \int_{0}^{1} d u C_{1}^{1 / 2}(2 u-1) \widetilde{\psi}_{3 \sigma}^{\mathrm{SR}}(\omega, u) \equiv 1
$$


We note that the expansion with respect to the Gegenbauer polynomials $C_{n}^{1 / 2}(2 u-1)$ is more suitable for these LCDAs. This is motivated by the analysis of the energetic $\pi$ meson for which the twist-3 $\phi_{p}(u)$ and twist- $4 g_{\pi}(u)$ LCDAs are described by the Gegenbauer polynomials $C_{n}^{1 / 2}(2 u-1)$ [31]-[33].

Numerical estimates for the $\Lambda_{b^{-}}$and $\Xi_{b}$-baryon moments based on the QCD sum rules are presented in Table 3. The moments $\left\langle\omega^{-1}\right\rangle,\left\langle\omega^{-1} C_{n}^{3 / 2}\right\rangle$, and $\left\langle\omega^{-1} C_{n}^{1 / 2}\right\rangle$ are dimensional with the table entries given in units of $\mathrm{GeV}^{-1}$, while $\left\langle C_{n}^{3 / 2}\right\rangle$ and $\left\langle C_{n}^{1 / 2}\right\rangle$ are dimensionless. As explained above, the errors correspond to the variation of the parameter $A(B=1-A)$ while the values of the other input parameters are taken from Table 2 and kept fixed at their central values. We note that integral $(60)$ with $\widetilde{\psi}_{3 \sigma}^{\mathrm{SR}}(\omega, u)$ for the $\Xi_{b}$ baryon becomes nonzero because the $S U(3)_{F}$ symmetry is broken, but it is numerically small:

$$
\int_{0}^{2 s_{0}} \omega d \omega \int_{0}^{1} d u \widetilde{\psi}_{3 \sigma}^{\mathrm{SR}}(\omega, u)=-0.0049_{-0.0037}^{+0.0049}
$$

The same also holds for the $\left\langle\omega^{-1}\right\rangle$ moment, as follows from Table 3.

Table 3

\begin{tabular}{|c|c|ccccccc|}
\hline$H_{Q}$ & $t$ & $\left\langle\omega^{-1}\right\rangle$ & $\left\langle C_{1}^{3 / 2}\right\rangle$ & $\left\langle\omega^{-1} C_{1}^{3 / 2}\right\rangle$ & $\left\langle C_{2}^{3 / 2}\right\rangle$ & $\left\langle\omega^{-1} C_{2}^{3 / 2}\right\rangle$ & $\left\langle C_{3}^{3 / 2}\right\rangle$ & $\left\langle\omega^{-1} C_{3}^{3 / 2}\right\rangle$ \\
\hline$\Lambda_{b}$ & 2 & $1.65_{-0.47}^{+0.91}$ & 0 & 0 & $1.00_{-1.03}^{+0.54}$ & $0.61_{-1.45}^{+0.76}$ & 0 & 0 \\
$\Xi_{b}$ & 2 & $1.46_{-0.34}^{+0.54}$ & $0.10_{-0.06}^{+0.10}$ & $0.08_{-0.05}^{+0.07}$ & $1.15_{-0.98}^{+0.61}$ & $0.86_{-1.10}^{+0.68}$ & $-0.02_{-0.52}^{+0.32}$ & $-0.10_{-0.38}^{+0.24}$ \\
\hline \hline$H_{Q}$ & $t$ & $\left\langle\omega^{-1}\right\rangle$ & $\left\langle C_{1}^{1 / 2}\right\rangle$ & $\left\langle\omega^{-1} C_{1}^{1 / 2}\right\rangle$ & $\left\langle C_{2}^{1 / 2}\right\rangle$ & $\left\langle\omega^{-1} C_{2}^{1 / 2}\right\rangle$ & $\left\langle C_{3}^{1 / 2}\right\rangle$ & $\left\langle\omega^{-1} C_{3}^{1 / 2}\right\rangle$ \\
\hline & $3 \mathrm{~s}$ & $2.16_{-0.36}^{+0.70}$ & 0 & 0 & $-0.032_{-0.041}^{+0.022}$ & $-0.29_{-0.27}^{+0.14}$ & 0 & 0 \\
$\Lambda_{b}$ & $3 \sigma$ & 0 & 1 & $1.54_{-0.22}^{+0.14}$ & 0 & 0 & $-0.034_{-0.021}^{+0.034}$ & $-0.027_{-0.017}^{+0.027}$ \\
& 4 & $2.84_{-0.46}^{+0.88}$ & 0 & 0 & $-0.108_{-0.018}^{+0.035}$ & $-0.41_{-0.15}^{+0.08}$ & 0 & 0 \\
\hline & $3 \mathrm{~s}$ & $1.94_{-0.21}^{+0.33}$ & $0.11_{-0.05}^{+0.09}$ & $0.075_{-0.047}^{+0.077}$ & $1.05_{-0.23}^{+0.14}$ & $1.01_{-0.46}^{+0.28}$ & $-0.014_{-0.032}^{+0.051}$ & $-0.117_{-0.005}^{+0.002}$ \\
$\Xi_{b}$ & $3 \sigma$ & $0.0019_{-0.0019}^{+0.0014}$ & 1 & $1.37_{-0.14}^{+0.11}$ & $0.057_{-0.057}^{+0.043}$ & $0.098_{-0.098}^{+0.075}$ & $1.11_{-0.35}^{+0.46}$ & $1.55_{-0.32}^{+0.24}$ \\
& 4 & $2.73_{-0.35}^{+0.61}$ & $0.12_{-0.05}^{+0.09}$ & $0.05_{-0.05}^{+0.09}$ & $0.55_{-0.11}^{+0.18}$ & $0.99_{-0.09}^{+0.16}$ & $-0.043_{-0.015}^{+0.025}$ & $-0.18_{-0.03}^{+0.02}$ \\
\hline
\end{tabular}

We propose simple models for the baryon LCDAs at the low scale $\mu=1 \mathrm{GeV}$ :

$$
\begin{aligned}
& \widetilde{\psi}_{2}(\omega, u)=\omega^{2} u(1-u) \sum_{n=0}^{2} \frac{a_{n}^{(2)}}{\epsilon_{n}^{(2)}{ }^{4}} C_{n}^{3 / 2}(2 u-1) e^{-\omega / \epsilon_{n}^{(2)}}, \\
& \widetilde{\psi}_{3 s}(\omega, u)=\frac{\omega}{2} \sum_{n=0}^{2} \frac{a_{n}^{(3)}}{\epsilon_{n}^{(3)^{3}}} C_{n}^{1 / 2}(2 u-1) e^{-\omega / \epsilon_{n}^{(3)}}, \\
& \widetilde{\psi}_{3 \sigma}(\omega, u)=\frac{\omega}{2} \sum_{n=0}^{3} \frac{b_{n}^{(3)}}{\eta_{n}^{(3)^{3}}} C_{n}^{1 / 2}(2 u-1) e^{-\omega / \eta_{n}^{(3)}}, \\
& \widetilde{\psi}_{4}(\omega, u)=\sum_{n=0}^{2} \frac{a_{n}^{(4)}}{\epsilon_{n}^{(4)^{2}}} C_{n}^{1 / 2}(2 u-1) e^{-\omega / \epsilon_{n}^{(4)}},
\end{aligned}
$$


where $C_{0}^{1 / 2}(2 u-1)=C_{0}^{3 / 2}(2 u-1)=1$. The zeroth-order Gegenbauer moments are defined as $a_{0}^{(t)} \equiv 1$, which follows from the normalization

$$
\int_{0}^{\infty} \omega d \omega \int_{0}^{1} d u \widetilde{\psi}_{t}(\omega, u) \equiv 1,
$$

where $t=2,3 s, 4$. In constructing the models for the LCDAs, we keep only two nonasymptotic terms in the Gegenbauer expansion. Moment values calculated based on the QCD sum rules were used to estimate values of the parameters in theoretical models (63)-(66) (see Table 4). The moments of interest expressed in terms of the model parameters introduced in Eqs. (63)-(66) are

$$
\begin{aligned}
& \left\langle\omega^{-1}\right\rangle_{t}^{H_{Q}}=\left\{\frac{1}{3 \epsilon_{0}^{(2)}}, \frac{1}{2 \epsilon_{0}^{(3)}}, \frac{b_{0}^{(3)}}{2 \eta_{0}^{(3)}}, \frac{1}{\epsilon_{0}^{(4)}}\right\}, \\
& \left\langle C_{1}^{3 / 2}\right\rangle_{t}^{H_{Q}}=\left\{\frac{9 a_{1}^{(2)}}{5}, \frac{a_{1}^{(3)}}{3}, \frac{b_{1}^{(3)}}{3}, \frac{a_{1}^{(4)}}{3}\right\}, \\
& \left\langle\omega^{-1} C_{1}^{3 / 2}\right\rangle_{t}^{H_{Q}}=\left\{\frac{3 a_{1}^{(2)}}{5 \epsilon_{1}^{(2)}}, \frac{a_{1}^{(3)}}{6 \epsilon_{1}^{(3)}}, \frac{b_{1}^{(3)}}{6 \eta_{1}^{(3)}}, \frac{a_{1}^{(4)}}{3 \epsilon_{1}^{(4)}}\right\}, \\
& \left\langle C_{2}^{3 / 2}\right\rangle_{t}^{H_{Q}}=\left\{\frac{18 a_{2}^{(2)}}{7}, \frac{a_{2}^{(3)}}{5}, \frac{b_{2}^{(3)}}{5}, \frac{a_{2}^{(4)}}{5}\right\}, \\
& \left\langle\omega^{-1} C_{2}^{3 / 2}\right\rangle_{t}^{H_{Q}}=\left\{\frac{6 a_{2}^{(2)}}{7 \epsilon_{2}^{(2)}}, \frac{a_{2}^{(3)}}{10 \epsilon_{2}^{(3)}}, \frac{b_{2}^{(3)}}{10 \eta_{2}^{(3)}}, \frac{a_{2}^{(4)}}{5 \epsilon_{2}^{(4)}}\right\} .
\end{aligned}
$$

Table 4

\begin{tabular}{|c|c|ccccc|}
\hline$H_{Q}$ & $t$ & $\varepsilon_{0}^{(t)}$ & $\varepsilon_{1}^{(t)}$ & $\varepsilon_{2}^{(t)}$ & $a_{1}^{(t)}$ & $a_{2}^{(t)}$ \\
\hline & 2 & $0.201_{-0.059}^{+0.143}$ & 0 & $0.551_{-0.356}^{+\infty}$ & 0 & $0.391_{-0.279}^{+0.279}$ \\
$\Lambda_{b}$ & 3 & $0.232_{-0.056}^{+0.047}$ & 0 & $0.055_{-0.020}^{+0.010}$ & 0 & $-0.161_{-0.207}^{+0.108}$ \\
& 4 & $0.352_{-0.083}^{+0.067}$ & 0 & $0.262_{-0.132}^{+0.116}$ & 0 & $-0.541_{-0.090}^{+0.173}$ \\
\hline & 2 & $0.228_{-0.061}^{+0.068}$ & $0.429_{-0.281}^{+0.654}$ & $0.449_{-0.473}^{+\infty}$ & $0.057_{-0.034}^{+0.055}$ & $0.449_{-0.380}^{+0.236}$ \\
$\Xi_{b}$ & 3 & $0.258_{-0.038}^{+0.031}$ & $0.750_{-0.093}^{+0.308}$ & $0.520_{-0.060}^{+0.229}$ & $0.339_{-0.160}^{+0.261}$ & $5.244_{-1.132}^{+0.696}$ \\
& 4 & $0.378_{-0.080}^{+0.065}$ & $2.291_{-0.842}^{+\infty}$ & $0.286_{-0.150}^{+0.130}$ & $0.039_{-0.018}^{+0.030}$ & $-0.090_{-0.021}^{+0.037}$ \\
\hline \hline$H_{Q}$ & $t$ & $\eta_{1}^{(t)}$ & $\eta_{2}^{(t)}$ & $\eta_{3}^{(t)}$ & $b_{2}^{(t)}$ & $b_{3}^{(t)}$ \\
\hline$\Lambda_{b}$ & 3 & $0.324_{-0.026}^{+0.054}$ & 0 & $0.633_{-0.0 ? ?}^{+0.0 ? ?}$ & 0 & $-0.240_{-0.147}^{+0.240}$ \\
$\Xi_{b}$ & 3 & $0.218_{-0.047}^{+0.043}$ & $0.877_{-0.152}^{+0.820}$ & $0.049_{-0.012}^{+0.005}$ & $0.037_{-0.019}^{+0.032}$ & $-0.027_{-0.027}^{+0.016}$ \\
\hline
\end{tabular}

In the limit of the exact $S U(3)_{F}$ symmetry, all the above functions have definite symmetry properties: $\widetilde{\psi}_{2}(\omega, u), \widetilde{\psi}_{3 s}(\omega, u)$, and $\widetilde{\psi}_{4}(\omega, u)$ are symmetric under the exchange $u \leftrightarrow 1-u$, while $\widetilde{\psi}_{3 \sigma}(\omega, u)$ is antisymmetric. As a result, only even Gegenbauer polynomials enter the symmetric model functions for the LCDAs $\left(a_{1}^{(t)}=0\right.$, where $\left.t=1,2,3\right)$, and odd polynomials are in the antisymmetric model function $\left(b_{0}^{(3)}=b_{2}^{(3)}=0\right)$. If we restrict ourself to only the isospin symmetry, then these symmetry conditions hold for the $\Lambda_{Q}$ baryon but not for $\Xi_{Q}$, because the $s$ quark contributes differently.

A detailed numerical analysis of the baryons with the $j^{p}=1^{+}$diquark will be presented in [8]. Here, we exemplify it by presenting the shape functions of the $J^{P}=1 / 2^{+}$baryons in Fig. 4 . The uncertainty in the LCDAs is mainly dominated by an arbitrariness $(0 \leq A \leq 1)$ in the choice of the local interpolating current. 

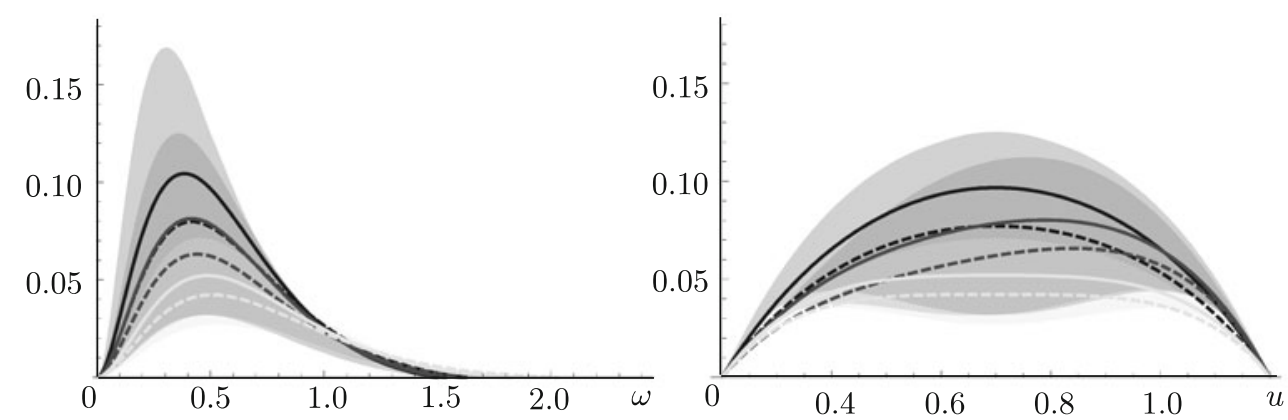

Fig. 4. Twist-2 LCDAs of $\Sigma_{b}, \Xi_{b}$, and $\Omega_{b}$ at the energy scales $\mu=1 \mathrm{GeV}$ (solid lines) and energy scale $\mu=2.5 \mathrm{GeV}$ (dashed lines) including the most conservative error from $A \in[0,1]$ (light shade).

\section{Conclusions}

We have constructed the total set of nonlocal light-ray operators for the ground-state heavy baryons with $J^{P}=1 / 2^{+}$and $J^{P}=3 / 2^{+}$in QCD in the heavy-quark limit. The matrix elements of these operators sandwiched between a heavy-baryon state and the vacuum determine LCDAs of different twists through the diquark current. With the QCD sum-rule method, we calculated several first moments of LCDAs using the nonlocal light-quark condensates. We proposed simple theoretical models for LCDAs and fitted their parameters based on the QCD sum-rule estimates. The $S U(3)_{F}$-breaking effects result in corrections of the order of $10 \%$.

Acknowledgments. One of the authors (A. Ya. P.) expresses his deep gratitude to the organizers of the XVI International Seminar on High Energy Physics "Quarks-2010" for their warm hospitality and thanks the Theory Group at DESY, where a major part of this work was done, for their kind hospitality.

This work was supported in part by the Federal Target Program "Scientific and Pedagogic Personnel of Innovative Russia" for 2009-2013 (State Contract No. P2323), the Ministry of Education and Science of the Russian Federation under the program "Development of the Scientific Potential of Higher Education" (Project No. 2.1.1/13011), the Russian Foundation for Basic Research (Grant No. 11-02-00394-a), and the Ministry of Education and Science of the Russian Federation in the framework of realization of the Federal Target Program "Scientific and Pedagogic Personnel of Innovative Russia" for 2009-2013 (Project No. P795).

\section{REFERENCES}

1. X.-G. He, T. Li, X.-Q. Li, and Y.-M. Wang, Phys. Rev. D, 74, 034026 (2006); arXiv:hep-ph/0606025v4 (2006).

2. A. Khodjamirian, T. Mannel, and N. Offen, Phys. Rev. D, 75, 054013 (2007); arXiv:hep-ph/0611193v2 (2006).

3. F. De Fazio, T. Feldmann, and T. Hurth, Nucl. Phys. B, 733, 1-30 (2006); Erratum, 800, 405-405 (2008); arXiv:hep-ph/0504088v4 (2005).

4. F. De Fazio, T. Feldmann, and T. Hurth, JHEP, 0802, 031 (2008); arXiv:0711.3999v2 [hep-ph] (2007).

5. W. Loinaz and R. Akhoury, Phys. Rev. D, 53, 1416-1424 (1996); arXiv:hep-ph/9505378v1 (1995).

6. F. Hussain, J. G. Körner, M. Krämer, and G. Thompson, Z. Phys. C, 51, 321-327 (1991).

7. P. Ball, V. M. Braun, and E. Gardi, Phys. Lett. B, 665, 197-204 (2008); arXiv:0804.2424v1 [hep-ph] (2008).

8. A. Ali, C. Hambrock, and A. Parkhomenko, "LCDAs of ground-state bottom baryons," (in preparation).

9. A. G. Grozin, Heavy Quark Effective Theory (Springer Tracts Mod. Phys., Vol. 201), Springer, Berlin (2004).

10. A. G. Grozin and O. I. Yakovlev, Phys. Lett. B, 285, 254-262 (1992); arXiv:hep-ph/9908364v1 (1999). 
11. S. Groote, J. G. Körner, and O. I. Yakovlev, Phys. Rev. D, 56, 3943-3954 (1997); arXiv:hep-ph/9705447v1 (1997).

12. E. V. Shuryak, Nucl. Phys. B, 198, 83-101 (1982).

13. Y.-B. Dai, C.-S. Huang, C. Liu, and C.-D. Lü, Phys. Lett. B, 371, 99-104 (1996); arXiv:hep-ph/9602242v1 (1996).

14. A. F. Falk, Nucl. Phys. B, 378, 79-94 (1992).

15. P. Ball, V. M. Braun, Y. Koike, and K. Tanaka, Nucl. Phys. B, 529, 323-382 (1998); arXiv:hep-ph/9802299v2 (1998).

16. E. Bagan, M. Chabab, H. G. Dosch, and S. Narison, Phys. Lett. B, 301, 243-248 (1993).

17. S. Groote, J. G. Körner, and O. I. Yakovlev, Phys. Rev. D, 55, 3016-3026 (1997); arXiv:hep-ph/9609469v1 (1996).

18. B. O. Lange and M. Neubert, Phys. Rev. Lett., 91, 102001 (2003); arXiv:hep-ph/0303082v1 (2003).

19. G. P. Lepage and S. J. Brodsky, Phys. Lett. B, 87, 359-365 (1979); Phys. Rev. D, 22, 2157-2198 (1980); A. V. Efremov and A. V. Radyushkin, Theor. Math. Phys., 42, 97-110 (1980); A. V. Efremov and A. V. Radyushkin, Phys. Lett. B, 94, 245-250 (1980).

20. A. G. Grozin and M. Neubert, Phys. Rev. D, 55, 272-290 (1997); arXiv:hep-ph/9607366v1 (1996).

21. V. M. Braun, D. Y. Ivanov, and G. P. Korchemsky, Phys. Rev. D, 69, 034014 (2004); arXiv:hep-ph/0309330v1 (2003).

22. S. V. Mikhailov and A. V. Radyushkin, JETP Lett., 43, 712-715 (1986).

23. S. V. Mikhailov and A. V. Radyushkin, Phys. Rev. D, 45, 1754-1759 (1992).

24. V. Braun, P. Górnicki, and L. Mankiewicz, Phys. Rev. D, 51, 6036-6051 (1995); arXiv:hep-ph/9410318v2 (1994).

25. A. G. Grozin, Internat. J. Mod. Phys. A, 10, 3497-3529 (1995); arXiv:hep-ph/9412238v2 (1994).

26. K. Nakamura et al. (Particle Data Group), J. Phys. G, 37, 075021 (2010).

27. X. Liu, H.-X. Chen, Y.-R. Liu, A. Hosaka, and S.-L. Zhu, Phys. Rev. D, 77, 014031 (2008); arXiv:0710.0123v2 [hep-ph] (2007).

28. R. Lewis and R. M. Woloshyn, Phys. Rev. D, 79, 014502 (2009); arXiv:0806.4783v4 [hep-lat] (2008).

29. J.-R. Zhang and M.-Q. Huang, Phys. Rev. D, 78, 094015 (2008); arXiv:0811.3266v1 [hep-ph] (2008).

30. K. G. Chetyrkin, A. Khodjamirian, and A. A. Pivovarov, Phys. Lett. B, 661, 250-258 (2008); arXiv:0712.2999v1 [hep-ph] (2007).

31. V. M. Braun and I. E. Filyanov, Z. Phys. C, 44, 157-166 (1989); Sov. J. Nucl. Phys., 50, No. 3, 511 (1989).

32. V. M. Braun and I. E. Filyanov, Z. Phys. C, 48, 239-248 (1990); Sov. J. Nucl. Phys., 52, 126-134 (1990).

33. P. Ball, JHEP, 9901, 010 (1999); arXiv:hep-ph/9812375v1 (1998). 